\title{
Resolution and Structural Transitions of Elongated States of Ubiquitin
}

\author{
Stormy L. Koeniger and David E. Clemmer \\ Department of Chemistry, Indiana University, Bloomington, Indiana, USA
}

\begin{abstract}
Electrospray ionization, combined with two-dimensional ion mobility spectrometry and mass spectrometry, is used to produce, select, and activate distributions of elongated ions, $[\mathrm{M}+$ $11 \mathrm{H}]^{11+}$ to $[\mathrm{M}+13 \mathrm{H}]^{13+}$, of ubiquitin. The analysis makes it possible to examine state-to-state transitions for structural types, and transition diagrams associated with the efficiencies of structural changes are presented. The +11 and +12 charge states can form four resolvable states while only one state is formed for $[\mathrm{M}+13 \mathrm{H}]^{13+}$. Some conformations, which appear to belong to the same family based on mobility analysis of different charge states, undergo similar transitions, others do not. Activation of ions that exist in low-abundance conformations, having mobilities that fall in between sharp peaks associated with higher abundances species, shows that the low-abundance forms undergo efficient ( $\sim 90$ to $100 \%)$ conversion into states associated with well-defined peaks. This efficiency is significantly higher than the $\sim 10$ to $60 \%$ efficiency of transitions of structures associated with well-defined peaks. The formation of sharp features from a range of low-intensity species with different cross sections indicates that large regions of conformation space must be unfavorable or inaccessible in the gas phase. These results are compared with several previous IMS measurements of this system as well as information about gas-phase structure provided by other techniques. (J Am Soc Mass Spectrom 2007, 18, 322-331) ( 2007 American Society for Mass Spectrometry
\end{abstract}

S ince the invention of soft ionization sources [1] for mass spectrometry (MS), a number of different methods have been devised to probe the structures of macromolecules in the absence of solvent. Studies of solvent-free proteins and peptides are important because of both fundamental and practical considerations. In the absence of solvation shells (or with minimal solvent), it is possible to extract kinetic and thermodynamic benchmarks about the formation of specific types of folds and tease out the influence of solvent-molecule and intramolecular factors in establishing conformation [2-11]. From a practical standpoint, a basic understanding of gas-phase macromolecular conformation is important because it is largely independent of the ion mass-to-charge $(\mathrm{m} / \mathrm{z})$ ratio. Therefore, assessing the conformations of gas-phase ions provides a means of complementing structural information obtained from MS measurements [12-20].

A number of groups have worked to combine ion mobility spectrometry (IMS) and MS with the aim of using differences in ion mobility to separate components of a mixture that would not be resolved by MS alone [21, 22]. The mobility of a macromolecular ion through a buffer gas depends on its charge and shape (average collision cross section with the buffer gas, $\Omega$ ). Recently, we have ex-

Published online November 2, 2006

Address reprint requests to Dr. D. E. Clemmer, Department of Chemistry, Indiana University, 800 E. Kirkwood Ave., Bloomington, IN 47405, USA. E-mail: clemmer@indiana.edu tended a hybrid IMS/MS instrument to include additional IMS dimensions leading to IMS-IMS/MS and IMS-IMSIMS/MS instrument designs [23-26]. One reason for developing these instruments is that it is possible to separate a distribution of initial conformations produced by an ion source, select a structural ion type (based on its mobility), and then activate the selected ions to induce transitions before characterizing the ions by separation through another drift region. By changing the structures of the ions, it is possible to resolve features that otherwise have identical mobilities $[23,26]$. Another motivation for developing this approach is that it makes it possible to examine state-to-state transitions. It is important to stress that it is currently unknown whether or not transitions observed in the gas phase are similar to transitions in solution. As shown below, the IMS-IMS approach makes it possible to select and follow transitions from many more starting points than can be done with techniques that are used to study structural changes in solution. The present paper demonstrates the ability to examine transition pathways in some detail; we do not attempt to relate these pathways to what is known in solution.

As one develops new IMS techniques (as well as other methods) it is important to revisit model systems, where some fundamental understanding of the nature of the system exists. In the present paper, we focus on the +11 to +13 charge states of ubiquitin ions produced by standard ESI conditions. Ubiquitin is a small, 76 amino acid protein that, under most ESI source conditions, favors the +5 to +13 charge states [27-32]. The 


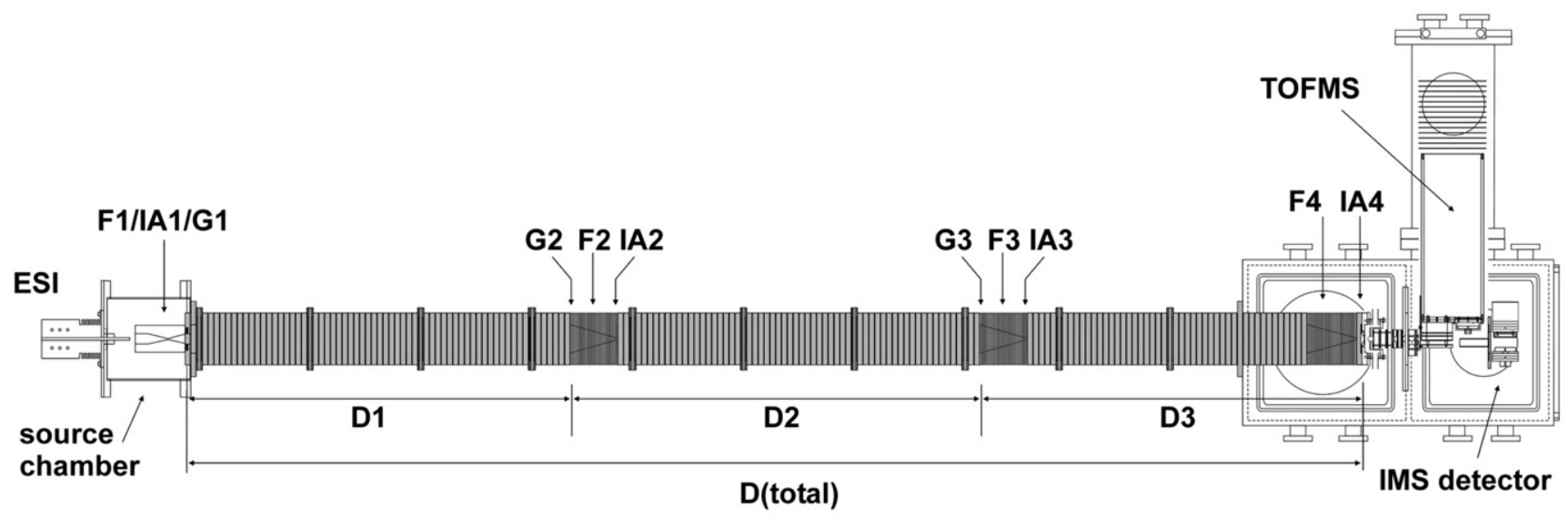

Figure 1. Schematic of IMS-IMS-IMS/MS instrument. A combination of ion gates (G1-G3), ion funnels (F1-F4), and ion activation regions (IA1-IA4) are incorporated into the drift tube assembly. For more detail on ion selection and activation, refer to text.

gas-phase structures of different charge states of ubiquitin have been investigated extensively, by a range of different methods including energy loss measurements [33,(34],@sotopic@xchange@ndథroton@ransfer@eaction measurements[27-29,@5-40],@nd@on@rapping@nd@on mobility@measurements $₫ 41, \mathbb{C} 2]$. CThere@re@everal@eports of the high-charge states (+11 to +13$)$, and evidence for multiple conformations of elongated structures exists. This evidence comes from several isotopic exchange@tudies $₫ 36-39] @ s @$ ell@s@ield@symmetric (FA)IMS@measurements $\$ 40,(43$, (44].đn@previous@vork,

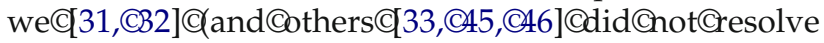
multiple structures for high-charge state ubiquitin ions, presumably because of the limited resolving power of early instruments.

In related work in which multiple conformations were resolved by IMS, we examined how $[\mathrm{M}+6 \mathrm{H}]^{6+}$, $[\mathrm{M}+7 \mathrm{H}]^{7+}$, and $[\mathrm{M}+8 \mathrm{H}]^{8+}$ evolve when ions are stored for varying times in a Paul geometry ion trap (over@0@nsథo@0@థ42]@nd@ttempted@o@orrelate@he results@o@sotopic@xchange@istributions $\$ 37] .($ he@rap work provides insight about how conformations evolve from initial states produced from the ESI droplet into structures that can exist for extended periods in the mass spectrometer. Although these are important benchmarksథonly@vailableథor@biquitin $\Phi 42] @ n d @ y t o-$ chrome@[47]),@he@xperimental@etup@s@imited(by@he low-resolution drift tube that was employed as well as difficulties in characterizing ion temperature in the trap [48].Che@ormer@imitation@ed@o@ur@ocus@n@ransitions involving the $[\mathrm{M}+7 \mathrm{H}]^{7+}$ and $[\mathrm{M}+8 \mathrm{H}]^{8+}$ that initially existed as compact and partially-folded forms and, after some time, evolved to elongated states. At the molecular level, the driving force for these transitions is the@eduction@f@epulsive@oulombic@nteractions $₫ 15$, 33,@49,@50].๑The@initial@and@final@structures@are@so different in shape that the transitions can be resolved, even with a low-resolution drift tube. This type of transition occurs for only a few charge states (intermediate states in which the attractive folding forces are nearly balanced by the repulsive interactions). Generally, high-charge states are not observed as compact structures; to date there has been no reports of structural transitions involving elongated families of conformations in high-charge states.

\section{Experimental}

\section{General}

Figure@1@shows@a@schematic@of@the@drift@ube@mass spectrometer used for these studies. Detailed descriptions@reథublished@lsewhereథ23];@nly@n@verview@f the instrument and experiment are presented here. Briefly, the instrument consists of a main drift tube that comprises three drift regions (D1 through D3), four ion funnels (F1 to F4), four ion gates (G1 to G3), and four ion activation regions (IA1 to IA4) as labeled in the figure. The instrument can be operated in a number of modes. For example, nested $t_{D}\left(t_{F}\right)$ datasets can be recorded by operating all drift regions and the (F2 to F4) funnels in transmission mode as described previously [23].@r,@t@s@ossible@o@elect@ons@f@pecified@nobilities at G2 or G3 as well as activate ions in the activation regions, as described in more detail below.

\section{Ion Funnels and Drift Regions}

Key to these experiments is the development of an integrated ion funnel/drift tube design. The funnel design is similar@o@ne@eveloped(By \$mith@nd@oworkers\$22]@nd the integrated funnel/drift tube instrument is similar

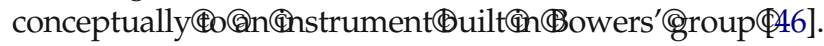
The F1 funnel is located at the entrance to the drift tube and functions to improve ion transmission and provides a means of accumulating the continuous ESI beam for pulsed experiments. The funnels (F2 and F3) are used to focus diffuse ion clouds back onto the axis of the drift tube; effectively this configuration makes high transmission,@ong@rift@ube@esigns@ossibleథ22,(23].@Addition- 
ally, the funnels provide a natural boundary for each of the three drift regions. The entire assembly [ $\mathrm{D}$ (total)] is $\sim 289 \mathrm{~cm}$ long, where D1 is $\sim 94 \mathrm{~cm}, \mathrm{D} 2$ is $\sim 100 \mathrm{~cm}$, and D3 is $\sim 95 \mathrm{~cm}$. The drift regions are operated at $9.0 \mathrm{~V} \cdot \mathrm{cm}^{-1}$. The axial fields of the funnels are: $17.0 \mathrm{~V} \cdot \mathrm{cm}^{-1}$ (F1); 11.0 $\mathrm{V} \cdot \mathrm{cm}^{-1}$ (F2 and F3); and $18.0 \mathrm{~V} \cdot \mathrm{cm}^{-1}$ (F4). Four RF generators built in-house provide voltage to the ion funnels: F1 is operated at $60 \mathrm{~V}_{p-p}(450 \mathrm{kHz}) ; \mathrm{F} 2$ and F3 are operated at $100 \mathrm{~V}_{p-p}(480 \mathrm{kHz})$; and F4 is operated at $70 \mathrm{~V}_{p-p}(450 \mathrm{kHz})$. The entire assembly is operated at $\sim 3.15$ torr helium.

\section{Ion Gating}

Ions can be gated at several locations in the instrument as

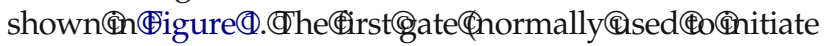
ion mobility measurements) is located at the exit of F1. Accumulated ions are released every $\sim 55 \mathrm{~ms}$ by lowering a repulsive field (for $100 \mu \mathrm{s}$ ) thus transmitting ions through G1. G2 is located at the entrance to F2, and serves to gate ions of a particular mobility into D2 in addition to initiating the second dimension of drift separation. The third gate is not utilized in the experiments presented here. The voltage applied to G2 is independent of the rest of the drift voltages and can be used to gate ions or set to maintain $11.0 \mathrm{~V} \cdot \mathrm{cm}^{-1}$ in F2 (normal transmission). For mobility selection, the repulsive voltage is lowered to the transmission voltage for $100 \mu \mathrm{s}$. The trap and release system is controlled by a pulse delay generator (model DG535, Stanford Research Systems, Inc., Sunnyvale, CA), and occurs at a defined time $t_{\text {delay }}$ after the initial drift pulse. The time that ions spend in each region can be determined empirically by gating the ion beam at each ion gate; under non-ion activation conditions (transmission/ selection), the total measured drift times are consistent with calculated values based on lengths and fields of each region. Much of the data shown below utilize a selection at G2. This is done by using a selection time $t_{\text {delay }}$ of $\sim 34 \%$ of the total measured drift time. For product ions (or new conformations) produced upon activation of a mobilityselected distribution, ion drift times are also corrected for the time of the selected ions to travel through F2 before activation.

\section{Ion Activation}

Ions are activated at IA2, a region that is made up of the last lens of F2 and the first lens of D2. The last lens of F2 has no RF field component, and the first lens of D2 has a grid across it to prevent RF penetration into D2. The voltages defined for IA2 refer to the voltage drop between these two electrodes. If no voltage is specified, the field through IA2 is $11 \mathrm{~V} \cdot \mathrm{cm}^{-1}$, the same as the dc component associated with the $\mathrm{F} 2$ region.

\section{Ion Formation}

A stock solution, containing ubiquitin (bovine, Sigma, $90 \%$ purity) was diluted to a $2.3 \times 10^{-5} \mathrm{M}$ concentration in a 49:49:2 (\% volume) solution of water:acetonitrile: acetic acid. Ions were produced by sending the solution through a pulled capillary tip $(75 \mu \mathrm{m}$ i.d. $\times 360 \mu \mathrm{m}$ o.d.), at a flow rate of $0.25 \mu \mathrm{L} \cdot \mathrm{min}^{-1}$, controlled by a syringe pump (KD Scientific, Holliston, MA). The pulled capillary was held at a DC bias $2.2 \mathrm{kV}$ above the drift voltage. A PEEK microtee was used to couple the capillary tip, the syringe, and a platinum electrode.

\section{Cross Section Distributions}

In the present study it is useful to convert drift time distributions directly into cross section distributions. This@s@ccomplished@sing@he@elation\$51]

$$
\Omega=\frac{(18 \pi)^{1 / 2}}{16} \frac{z e}{\left(k_{b} T\right)^{1 / 2}}\left[\frac{1}{m_{l}}+\frac{1}{m_{B}}\right]^{1 / 2} \frac{t_{d} E}{L} \frac{760}{P} \frac{T}{273.2} \frac{1}{N}
$$

where $t_{d}, E$, and $L$ are the drift time, electric field, and drift length, respectively, for the region of interest; $T$ and $P$ are the temperature and pressure of the buffer gas, respectively; $z e$ is the ion's charge; $k_{b}$ is Boltzmann's constant; $m_{I}$ and $m_{B}$ are masses of the ion and buffer gas, respectively; and $N$ is the neutral number density. Because the current drift tube is somewhat more complicated than earlier designs, we start each series of experiments by recording the drift time associated with the D1 region. This region is designed to have a very uniform field (and the pressure and length are known). Effectively, the defined delay time associated with the ion gate allows only a narrow range of ions (with defined mobilities or cross sections) to pass into the remaining portions of the instrument. Thus, under nonactivating conditions, there is always an internal check of the measured cross sections through the remaining portion of the instrument. Thus, cross sections measurements are highly accurate and precise. Comparison of a cross section measurement with this system with a value measured by another well characterized instrument usually shows agreement to within $2 \%$ (relative uncertainty) [23].CThe@recision@f@hese@neasurements@s@ven@etter with any two measurements (taken over a period of several months) agreeing to within $1 \%$. The primary source of uncertainty associated with day to day measurements is associated with the buffer gas temperature along the drift tube axis, which probably changes with variation in the room temperature.

\section{Results and Discussion}

\section{General Description of Cross Section Distributions for Ubiquitin Charge States}

Figure@@hows@he@ross@ection@istributions@hat@re obtained upon IMS separation of the +5 to +13 charge states ubiquitin. These data are displayed on a scale that indicate regions associated with our previous definitions of compact, partially-folded, and elongated states [31].đhe@mall@eak $థ$ shaded@n@ray)@bserved@or@he distribution labeled as +5 actually corresponds to a small population of $[2 \mathrm{M}+10 \mathrm{H}]^{10+}$ ions (and is not 


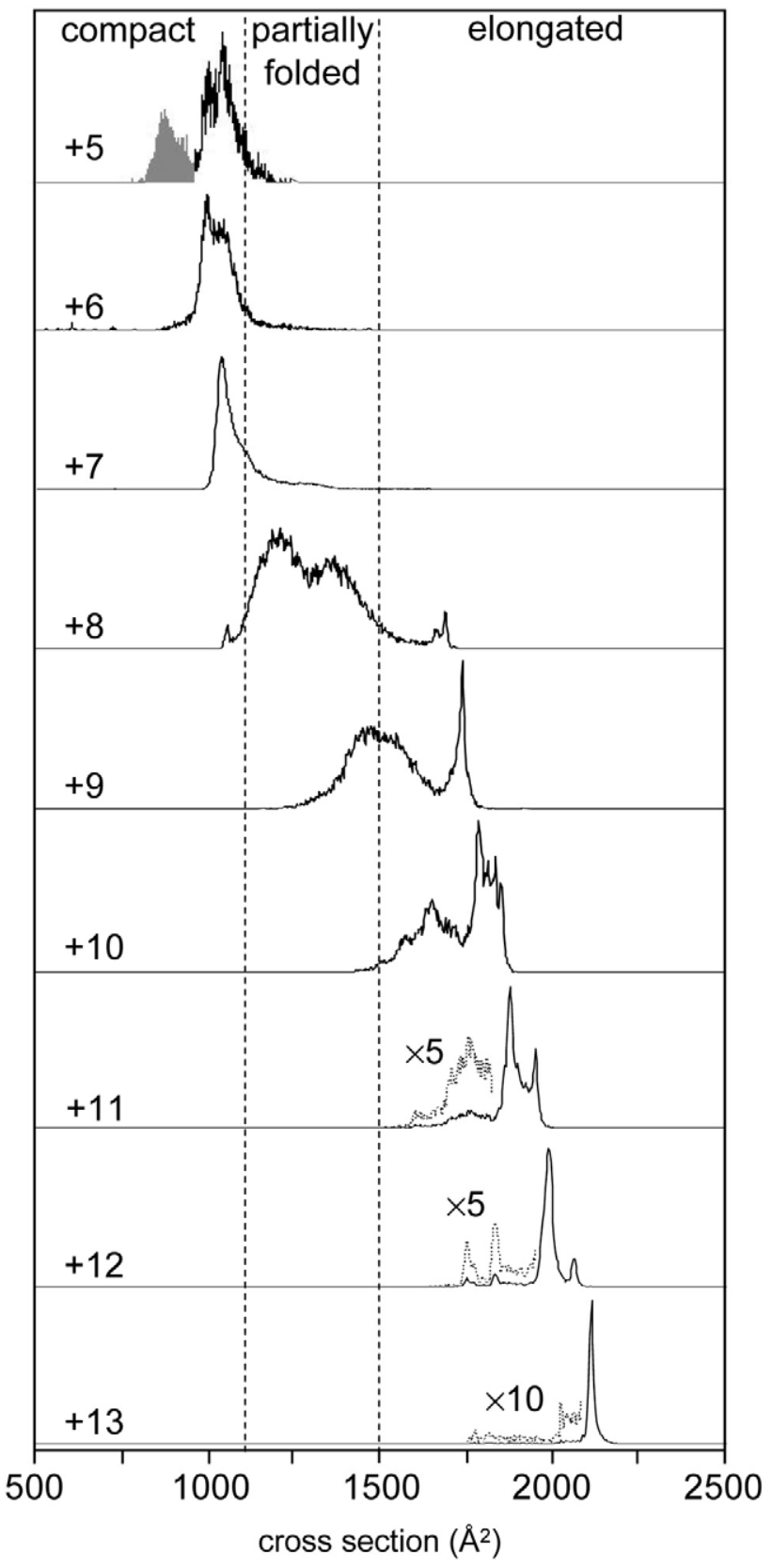

Figure 2. Cross section distributions for the +5 to +13 charge states of electrosprayed ubiquitin ions. Regions corresponding to compact, partially-folded, and elongated conformations are indicated. The $[2 \mathrm{M}+10 \mathrm{H}]^{10+}$ (filled gray) for the +5 charge state is identified from a small family of dimers observed in the $t_{D}\left(t_{F}\right)$ distribution. Low intensity features of the +11 to +13 charge states are shown magnified (dotted lines). Distributions are obtained by taking slices from a $t_{D}\left(t_{F}\right)$ dataset.

discussed more here). Essentially, all of the main features that are observed are consistent with our previous

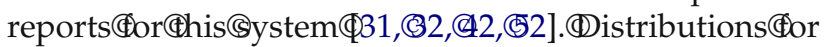
the +5 to +7 charge states are dominated by compact ions; the +8 charge state displays a peak in the compact region at $1063 \AA^{2}$, a broad distribution of partiallyfolded ions (in a distribution that shows two features) as well as two small peaks at 1670 and $1695 \AA^{2}$ (elongated states). Similarly the distribution for the +9 charge state displays a range of partially-folded ions that extends into the region defined as elongated, and a single sharp peak at $1733 \AA^{2}$ (corresponding to elongated ions). The distributions for the +10 to +13 charge states show that these ions have elongated conformations.

Although, we consider these results to be typical for this system, there are some subtle differences with previous@MS@istributions $₫ 41, \mathbb{4} 2] \oplus h a t @$ robably@rise from differences in source configurations, storage time, and higher resolution. These subtle differences, however, do not influence our results in the studies reported here. Some of these differences are consistent with reports by others. For example, the +10 to +12 charge states show evidence for multiple stable elongated forms (not reported by us previously, but consistent

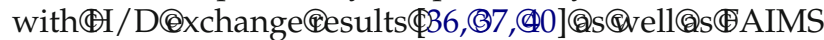

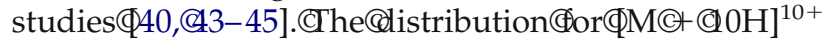
shows a broad unresolved structure (from $\sim 1490$ to $\sim 1780 \AA^{2}$; although this distribution is broad, there are two reproducible features with maxima at $\sim 1590$ and $\sim 1660 \AA^{2}$. Additionally, several sharp peaks are observed for even larger cross section ions. Two (at $\sim 1790$ and $1850 \AA^{2}$ ) are reproducible; the region between these peaks varies substantially between measurements.

The distribution for $[\mathrm{M}+11 \mathrm{H}]^{11+}$ shows a similar (although lower-abundance) population of unresolved ions; additionally there are two sharp peaks at 1879 and $1953 \AA^{2}$. The ratios of these peaks are similar to the ratios observed for the similar reproducible sharp peaks in the $[\mathrm{M}+10 \mathrm{H}]^{10+}$ distribution. The $[\mathrm{M}+12 \mathrm{H}]^{12+}$ distribution is dominated by a narrow peak at $1998 \AA^{2}$. Several smaller, well-defined peaks at 1767,1845 , and $2072 \AA^{2}$ are also observed. A close examination of the region between these peaks shows that there is a small (non-zero) baseline, associated with a population of structures between peaks. We note this because in this way the +12 charge state also displays evidence for a broad unresolved feature (similar to $[\mathrm{M}+10 \mathrm{H}]^{10+}$ and $\left.[\mathrm{M}+11 \mathrm{H}]^{11+}\right)$. The distribution for $[\mathrm{M}+13 \mathrm{H}]^{13+}$ is dominated by a single sharp peak at $2115 \AA^{2}$, this cross section is very similar to the value we calculated and reported previously for a near-linear structure $\Omega$ (linear)

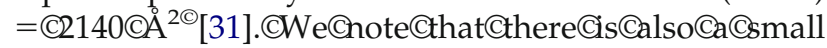
shoulder, associated with the lower cross section ions.

It is worthwhile to consider the origin of broad features. One might anticipate that as solvent is removed from a protein, there may be conditions under which some elements of structure are preserved into the gas-phase@2,(C8]; Chowever,@upon@ctivation@in@he@bsence of solvent one might expect that many similar structures may exist and there may be few favored states (if any). A pronounced example of where this may be the case can be observed from the distribution for the +8 charge state. In this case, where the repulsive Coulombic and attractive folding interactions are closely balanced, the distribution is dominated by broad distributions of unresolved features that span a 

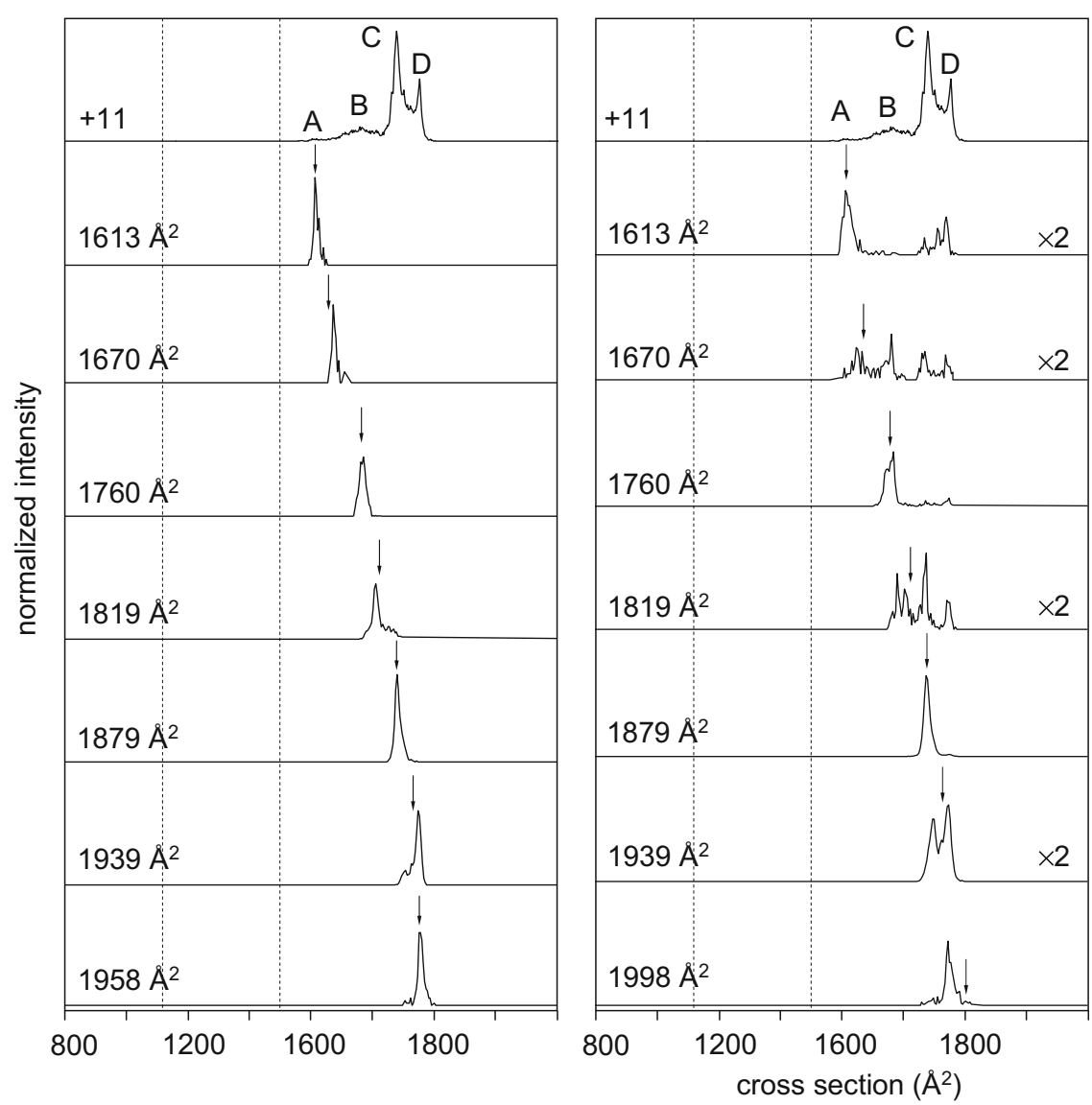

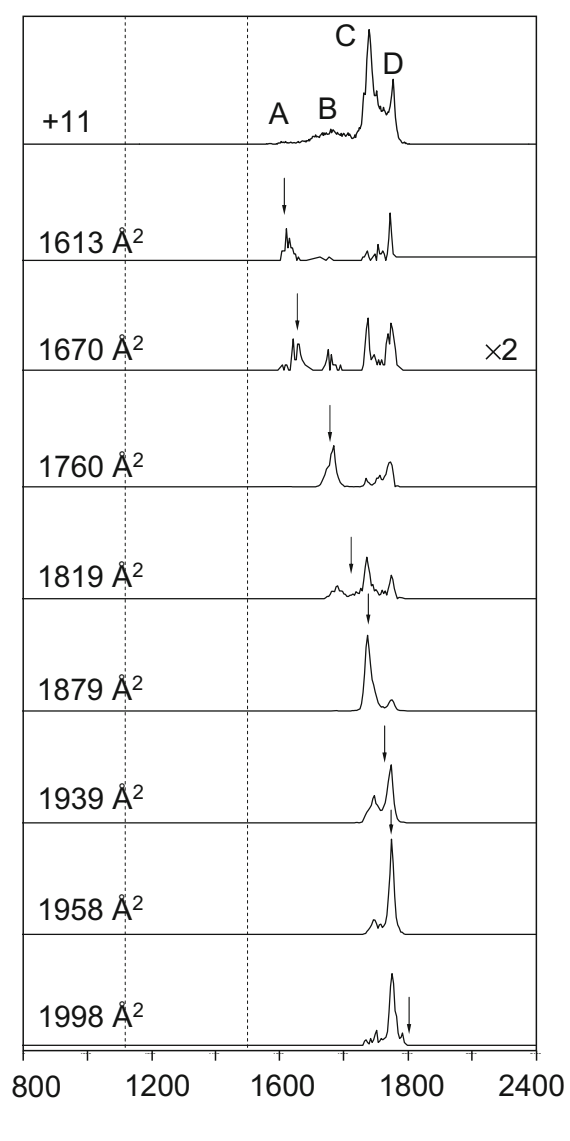

Figure 3. Cross section distributions for the +11 charge state of ubiquitin obtained upon selection and activation of specific mobility regions at G2 and IA2, respectively. Four stable conformations (labeled A-D) are observed within the initial distribution of ions produced by ESI (top). Mobilityselected ion distributions (with no activation) indicate the presence of stable and unstable ion populations (left). Activation of these ions with $80 \mathrm{~V}$ (middle) and $100 \mathrm{~V}$ (right) produces a new distribution that is unique to the selected ions. Distributions are labeled according to the cross section that was selected (indicated by the arrow in the distribution), and dashed lines delineate the regions corresponding to the compact, partially-folded, and elongated conformations. Distributions are obtained by taking slices from $t_{D}\left(t_{F}\right)$ data sets. See text for discussion.

large cross section range; there is ion signal across the entire $\sim 1050$ to $1700 \AA^{2}$ range. As the charge state increases, the broad unresolved feature shifts to higher cross sections (consistent with the increase in repulsive forces) and decreases in abundance.

\section{Selection and Activation of Ions}

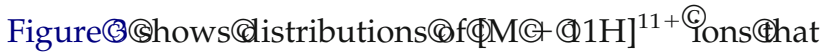
are obtained upon selection of specific mobility regions (at G2) and activation at two activation voltages (80 and $100 \mathrm{~V})$ at IA2. Several aspects of these data are noteworthy. The first involves the distributions associated with the selected ions with no activation. For example, selection of the largest peak $\left(\Omega=1879 \AA^{2}\right)$ leads to a distribution that is dominated by a single narrow peak (also having $\Omega=1880 \AA^{2}$ ). On the other hand, selection of ions at $\Omega=1819 \AA^{2}$ leads to a distribution in which the main peak has shifted slightly (to a value of $\Omega=$ $1810 \AA^{2}$; additionally, shoulders corresponding to slightly larger and smaller cross section ions are ob- served. In the former case $\left(\Omega=1879 \AA^{2}\right)$, apparently a relatively stable ion was selected; in the latter case, apparently even without activation we observe the onset of structural transitions (i.e., the distribution that was selected was relatively unstable). From this, we characterize ions present in the initial distribution as stable $\left[\Omega(\right.$ selected $)=1613,1760,1879$, and $1958 \AA^{2}$. All other selected regions (centered away from these stable regions) show evidence of structural transitions (with no activation). Of the four stable ions, only two $(\Omega=$ 1879 and $1953 \AA^{2}$ ) appear as resolved peaks in the IMS distribution; and these peaks are not baseline resolved.

A second notable aspect of these data involves the products that are produced upon activation. That is, the +11 charge state appears to primarily favor the same product ions. For example, activation of $\Omega$ (selected) $=$ $1613 \AA^{2}$ leads to peaks at $\Omega=1620$ and $1949 \AA^{2}$. Several other examples include: $\Omega$ (selected) $=1670 \AA^{2}$ leads to $\Omega=1628,1770,1876$, and $1949 \AA^{2}$; and $\Omega$ (selected) $=$ $1819 \AA^{2}$ leads to $\Omega=1783,1876$, and $1952 \AA^{2}$. Other selections,@some@shown@in@Figure@3,@lead@to@these 

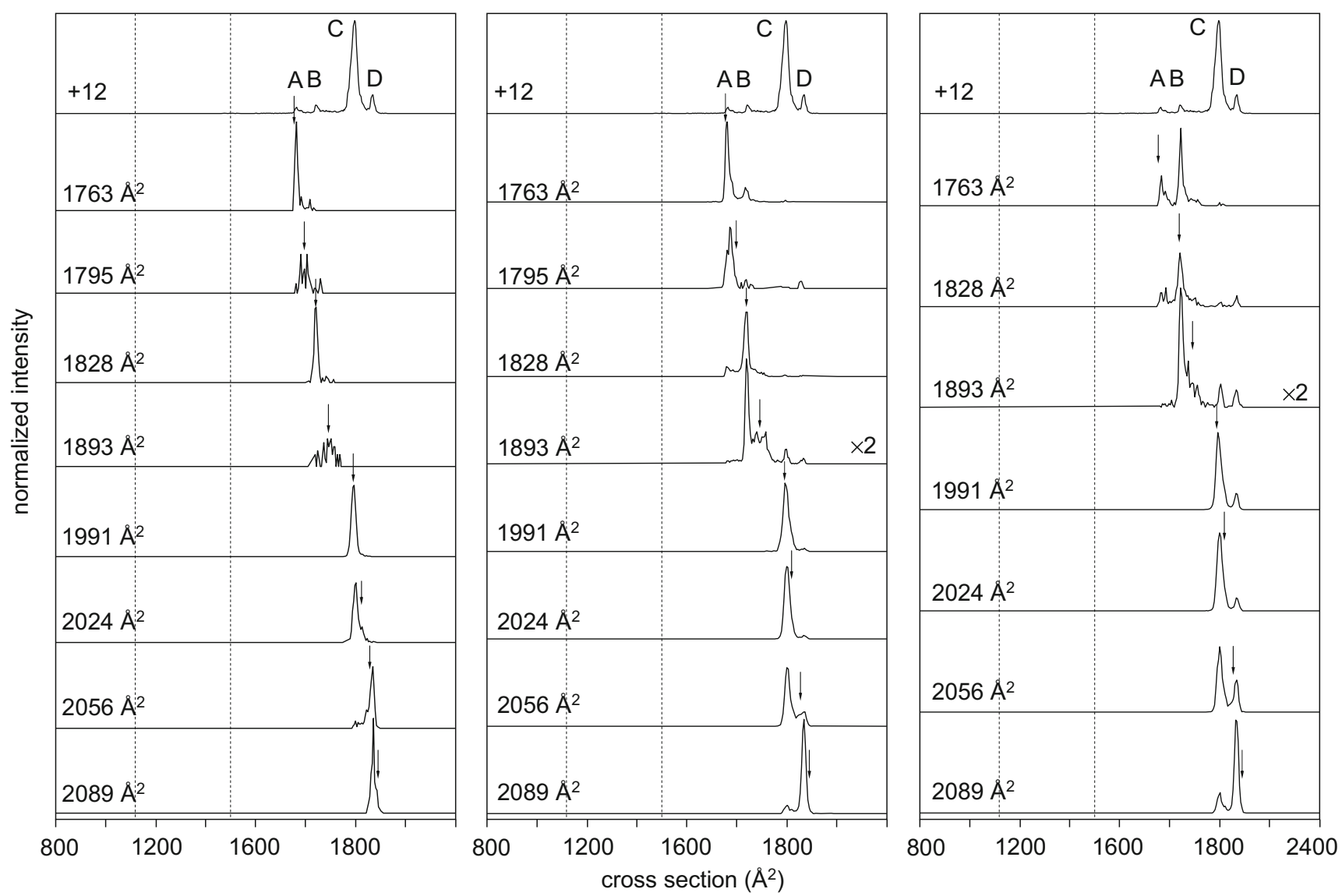

Figure 4. Cross section distributions for the +12 charge state of ubiquitin obtained upon selection at G2 (left) and activation at IA2 with $80 \mathrm{~V}$ (middle) and $100 \mathrm{~V}$ (right). Four stable conformations (labeled A-D) are observed within the initial distribution of ions produced by ESI (top). Details of the figure are@he@ame@s@hose@nథFigure@3.(See@ext@for@iscussion.

products. Overall, apparently there are primarily four different conformations identified through selection and activation of different regions within the IMS distribution, which we have labeled A through D in FigureC8.CAlthough@our@esolved@tructures@re@more than we reported previously (and more than has been reported in some other studies), this value is consistent with a measurement of the +11 charge state of ubiquitin obtained@sing@AIMS $\Phi 43]$.

Finally, we note that the product distribution depends strongly upon the initial selection. Although peak@@ominates@he@MS@istribution $\Phi$ Figure@2), (t) not formed significantly from $\Omega$ (selected) $=1613$ or $1760 \AA^{2}$ precursors. It is, however, observed upon activation of all of the other $\Omega$ (selected) regions that are shown.

Figure@4@hows@n@inalogous@nalysis@to@be@ead three times quickly) of the +12 charge state. The three primary conclusions that were drawn from the data in Figure@B@are@lso@pparent@n@hese@distributions:@1) selected ions can be identified as stable or unstable; (2) activation of any selected region leads to a limited number of favored products (also four primary products, as in the +11 charge state analysis); and (3) the final distribution of products is dependent upon the initial selected state. In corroboration of the first conclusion, two examples of selections involving stable distributions of states are $\Omega$ (selected) $=1828$ and 1991; examples of selected distributions that show evidence for spontaneous conversion to new states include $\Omega$ (selected $)=1795$ and $2024 \AA^{2}$. Similar to the +11 charge state apparently there are primarily four different product states that are produced by the range of different selections (also labeled A through D). Finally, examination of the distribution obtained upon activation of the $\Omega($ selected $)=1763 \AA^{2}$ leads to formation of primarily B, but not $C$, even though this peak dominates the IMS distribution@hown@nథigure@.

Figureฐ(@hows@he@ame@nalysisథor@he@ 13@harge state@istribution.@n@ontrast@o@he@ata@n@igures $\mathbb{C}$ and@↔ the distributions for the +13 charge state show that although a range of different structures can be selected [away from the primary peak $(\Omega=2115 \AA 2)$ and including regions at higher cross section where there appears to be no ion signal] upon activation only a single peak is favored. We note that the observed transitions of the low abundance ions to the primary peak position indicate that these ions are not chemical noise. The observation of a single peak for the +13 

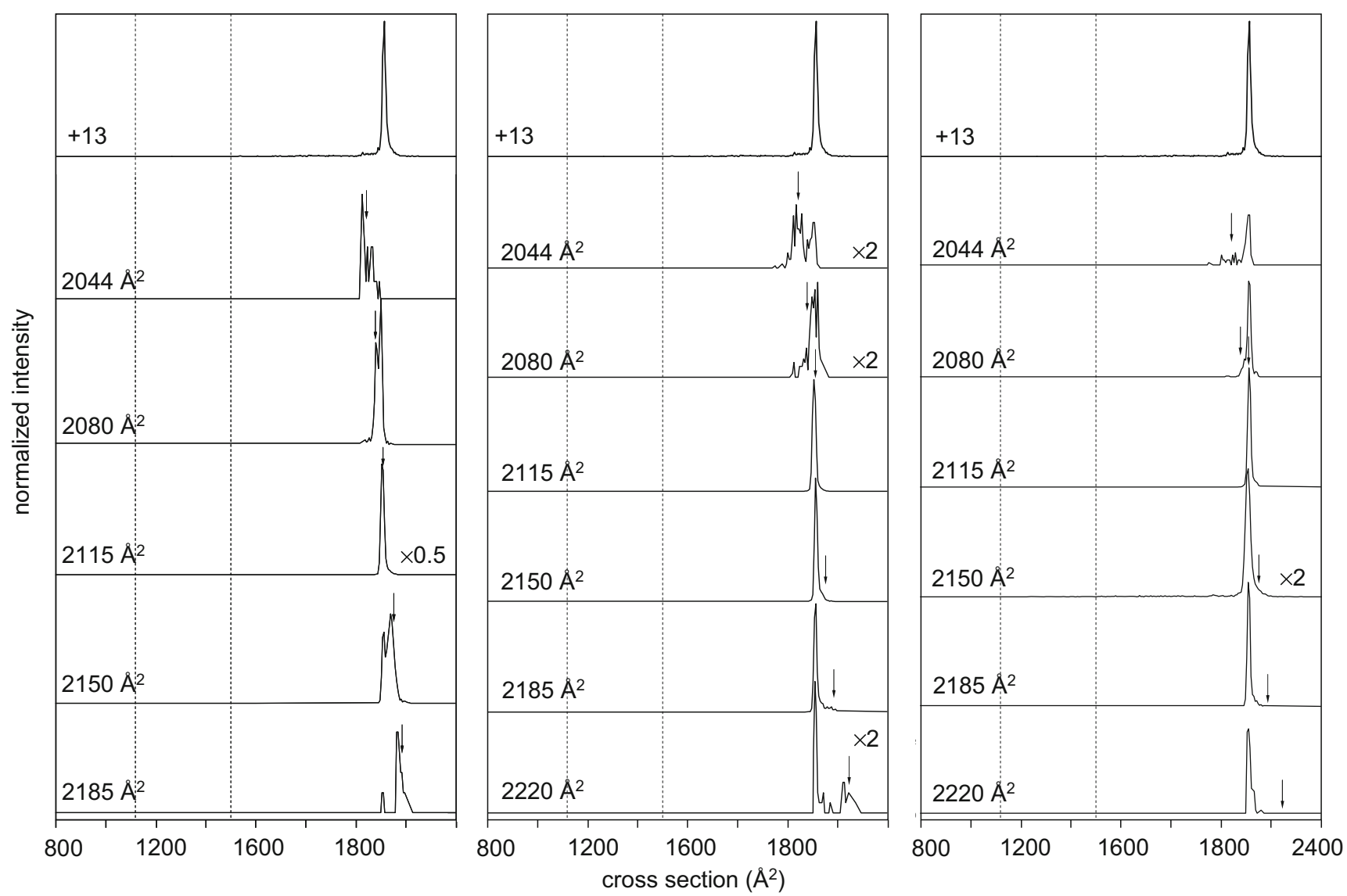

Figure 5. Cross section distributions for the +13 charge state of ubiquitin obtained upon selection at G2 (left) and activation at IA2 with $80 \mathrm{~V}$ (middle) and $100 \mathrm{~V}$ (right). The initial distribution of ions

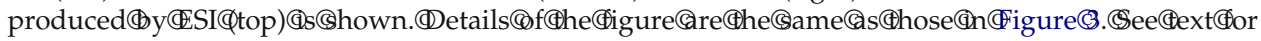
discussion.

charge@state@is@consistent@with@a@number@of@other measurements, @including@heđFAIMS@analysis@40,@43, 45].@We@do@not@show@ita@for@he@+10@harge@state; however, $₫$ he@esults $₫$ or $₫$ hese $($ ons $@$ re@imilar@o@he $₫ 11$ and@1 12@harge@tates.

\section{Transition Diagrams for Elongated States of Ubiquitin}

Figure@@hows@@ummary@f@he@lata@resented@in Figures@3@o@s@s@ransition@iagrams.CThese@iagrams show@he@elative@fficiencies@ssociated@ith@he@tructural@hanges@f(both@table@nd@nstable@ons@dentified above.@We@egin@ur@nalysis@y@xamining@he@ransitions@f@table@ons $\$$ conformations $₫ a b e l e d @(\$ h r o u g h(\Phi)$

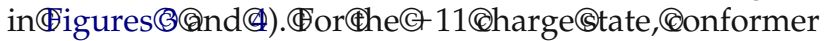
A@roduces@@ndథ®vith@elatively@ow@fficiencies@f 13@nd@29\%,@espectively.@A@oes@ot@roduce@B.@onformer@B)roduces@@nd@, (also@nefficiently@7@and 19\%,Crespectively),Cbut@B@also@does@not@produce@A. Conformer@@vill@nly@roduce@D@vith@ow@fficiency (6\%),@ndథ®ill@nlyథroduce@ $\$ 15 \%) .(T h e @ m o u n t @ f$ conformer@A, $\mathbb{C B}, \propto C$, Cand@C Cremaining@post@activation provides@nsight@bout@he@elative@tabilities@f@hese conformations.CFor@xample,@onformer@@@s@he@pre- ferred@conformation@with@94\%@remaining@post-activation,@hereas@nlyब58\%@f@onformer@, @ith@heథlowest cross@ection,@emains@ost@ctivation.@verall,@he@low efficiency@f@hese@ransitions@uggests@hat@hese@ion populations@conformers)@epresent@he@avorable@onformations@ampledథoth@rom $₫$ he@lectrospray@rocess and@hrough@ctivation.

A@ifferent@elationship@f@onformers@@@nd@@@re observed@for@the $(+12 @$ charge@state.CConformer@A produces@®ith@6\%@fficiency@nd@o@đesser@xtent

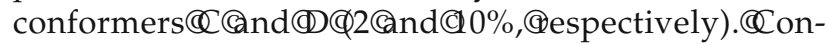
former@B@produces@A@with@22\%@efficiency@and@a smaller@mount@f@@ndథథ5@nd@\%,@espectively). Similar@o@he@+11@harge@tate,@onformer@@and( only@roduce@ech@ther@8@and@18\%,@espectively). Low@fficiency@ransitions@re@lso@bservedథor@hese conformers.

Based@n@he@ough@orrelation@hat@xists@etween mobility@nd@harge@tate,@ikely@he@ominant@eak@n

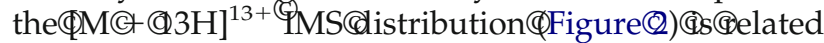
to@onformers@@r(®@f(4he@11@nd@ 12@harge@tates. This@conformer@does@not@produce@any@other@peaks upon@ctivation;@t@ppears@o@e@he@nly@ccessible state@bserved@ver $₫$ he甲recursor@ange@hat@ouldథe studied. 

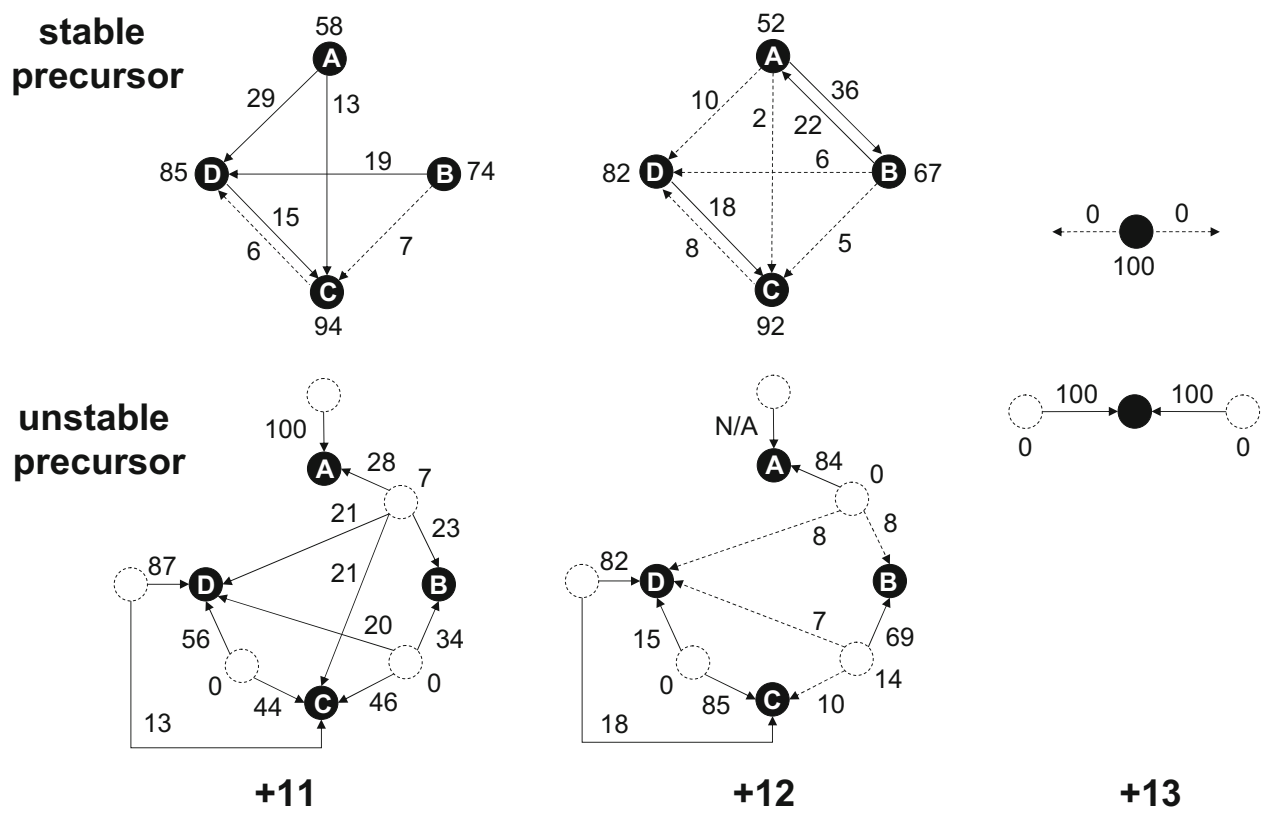

Figure 6. Transition diagrams showing the \% efficiency of stable (top, labeled A-D) and unstable (bottom, dashed circles) precursor transitions upon activation at IA2. The \% efficiency is the average of the relative abundance of each conformer formed (labeled A-D) upon activation at 80 and $100 \mathrm{~V}$. Transitions of low efficiency $(\leq 10 \%)$ are indicated by dotted lines. Unstable ion populations selected at higher mobility than conformer $\mathrm{A}$ and lower mobility than conformer $\mathrm{D}$ are shown above and to the left, respectively, of the solid circles A-D for the +11 and +12 . Selection and activation of unstable ion populations at higher and lower mobility for the +13 are also shown. See text for discussion.

Regions of unstable ions (regions not defined as A through@D)Care@also@eharacterized@in@Figure@6.CFirst, consider the $[\mathrm{M}+11 \mathrm{H}]^{11+}$. Selection and activation of ions between conformers $\mathrm{A}$ and $\mathrm{B}$ produce conformers A, B, C, and D, a highly efficient transition in which only $7 \%$ of the selected ions remain. Ions between conformers $B$ and $C$ as well as between $C$ and $D$ are $100 \%$ efficient at producing B, C, and D and C and D, respectively. Surprisingly, if ions are selected at lower mobility than D where there are no peaks (but there exists a very small continuous baseline signal), $87 \%$ of these ions produce D and 13\% produce C. Similarly, ions at higher mobility than conformer A produce conformer A with 100\% efficiency. Overall, the transition efficiency of ions between conformers does not favor any single transition.

For $[\mathrm{M}+12 \mathrm{H}]^{12+}$, transition trends are similar (to $\left.[\mathrm{M}+11 \mathrm{H}]^{11+}\right)$; however some differences are apparent (i.e., specific conformers are formed more favorably than others). This difference is not completely unexpected based on the different structural transitions observed for conformer $\mathrm{A}$ and $\mathrm{B}$; however, the ion population between $\mathrm{C}$ and $\mathrm{D}$ shows different efficiencies in the +11 and +12 charge states even though conformer $\mathrm{C}$ and D show similar efficiencies. This difference could arise due to slight variations in structure associated with each charge state. Ions selected at higher or lower mobility than conformer D of the +13 charge state form conformer D with $100 \%$ efficiency.

\section{Summary and Conclusions}

Structural transitions of elongated ubiquitin ions (formed by ESI) are examined using IMS-IMS/MS instrumentation. Within the +11 and +12 charge states, we observe four resolvable peaks associated with different conformations; for the +13 , we observe a single resolved peak. The results show that some regions within IMS distributions are stable; others are not. That is, upon selection of a stable ion population, a single narrow peak is observed with the peak maximum at the location of the selection; unstable ions are identified from peaks that shift and broaden-an indication of structural transitions. The timescales of the transitions that are observed vary, from those that must occur rapidly relative to the timescale of the measurement, i.e., $<50 \mu$ s (e.g., timescales associated with changes upon activation of some selected ions), to those that are much slower (e.g., on the $\sim 20$ to $25 \mathrm{~ms}$ timescale of the measurement such as is observed for unstable states without activation). The observation of both stable and unstable ion populations within IMS distributions may be related to remnants of structures that were accessible in solution.

In the gas phase, one might expect that many different conformations should be accessible upon activation (since the factors that govern folding evolved in solution); thus, one might expect that some selected states should lose structure in a random way when activated and, upon cooling, it seems reasonable that many 
different structures could be stabilized. We expect that this type of process would lead to broad IMS distributions, with few resolved peaks. We are surprised to find no evidence for this behavior in any region of the selected ions. In all of the selected regions that we examine for these high charge states, activation leads to relatively sharp peaks, indicating that all of the selected species appear to be capable of spontaneously finding (either with or without activation) highly favored structures (and that these structures exist over narrow regions of the available cross section space).

Taken together, these results require that there are a few specific conformations that are not only favored in the gas phase but are also accessible to a specific range of selected precursors. That is, the distribution of favored final states depends upon the conformation type that is selected. Transitions between states appear to be pathway dependent. To understand these processes, we developed several simple diagrams that illustrate how populations of selected regions evolve to new states upon@ctivation@Figure@).(These@ransition@iagrams indicate that the low mobility conformers ( $C$ and $D)$ of the +11 and +12 show very similar transitions and efficiencies, whereas the high mobility conformers (A and $B$ ) show different behavior for the +11 and +12 charge states. These results may indicate that although the cross section distributions between charge states suggest that these states are similar, this may not be the case (since the transitions between states appear to be relatively different). This type of difference can be resolved in a multidimensional measurement, but is missed in measurements where only one physical or chemical@roperty@s@haracterized@40].CThis@has@amifications about how conformer types are grouped. It is common to group conformations within charge states into families on the basis of their collision cross sections. If this were the only measure in which to relate gasphase conformers, then conformer A from $[\mathrm{M}+$ $11 \mathrm{H}]^{11+}$ would be grouped into a family with conformer A from $[\mathrm{M}+12 \mathrm{H}]^{12+}$ and likewise for conformers B through D. However, these results indicate that there are clear differences in the state-to-state transitions of conformers A and B in both charge states. The present results agree with a conclusion by Williams and coworkers from combined FAIMS/isotopic exchange studies, "grouping conformers into families based on a single physical property may over-simplify the complexity@f@hese@as-phase@onformers"@40].@We@note that it should be possible to incorporate isotopic exchange into IMS-IMS/MS measurements (allowing reactivity differences to be used for resolution of structure and pathway).

It is interesting to consider the future prospects of this technique to study and compare the transitions of gas-phase conformations to folding in solution. An advantage of the present approach compared to those in solution is that structural transitions of many different states can be studied directly (i.e., independently of the ensemble of conformations). Folding studies in solution are highly limited in this respect. With the exception of a few recent and high-profile studies [53-58],థolding@f@individual@tates@as@ot@eenథossible except in silico. In the present system, it remains unknown whether or not structures and transition pathways observed in the gas phase are related to those in solution; in future work, we will examine a system with well-characterized transitions in solution.

\section{Acknowledgments}

This work is supported by grants from the National Institutes of Health (AG-024547-02 and P41-RR018942), and the Analytical Node of the Indiana University METACyt initiative funded by the Lilly Endowment.

\section{References}

1. (a) Karas, M.; Hillenkamp, F. Laser desorption ionization of proteins with molecular masses exceeding 10,000 daltons. Anal. Chem. 1988, 60, 2299-2301. (b) Tanaka, K.; Waki, H.; Ido, Y.; Akita, S.; Yoshida, Y.; Yoshida, T. Protein and polymer analyses up to $\mathrm{m} / \mathrm{z} 100,000$ by laser ionization time-of-flight mass spectrometry. Rapid Commun. Mass Spectrom. 1988, 2, 151-153. (c) Fenn, J. B.; Mann, M.; Meng, C. K.; Wong, S. F.; Whitehouse, C. M. Electrospray Ionization for Mass Spectrometry of Large Biomolecules. Science 1989, 246, 64-71. (d) Takats, Z.; Wiseman, J. M.; Gologan, B.; Cooks, R. G. Mass Spectrometry Sampling Under Ambient Conditions with Desorption Electrospray Ionization. Science 2004, 306, 471-473

2. (a) Suckau, D.; Shi, Y.; Beu, S. C.; Senko, M. W.; Quinn, J. P.; Wampler, F. W.; McLafferty, F. W. Coexisting Stable Conformations of Gaseous Protein Ions. Proc. Natl. Acad. Sci. U.S. A. 1993, 90, 790-793. (b) Wood, T. D.; Chorush, R. A.; Wampler, F. M.; Little, D. P.; O'Connor, P. B.; McLafferty, F. W. Gas-Phase Folding and Unfolding of Cytochrome $c$ Cations. Proc. Natl. Acad. Sci. U.S.A. 1995, 92, 2451-2454.

3. Wolynes, P. G. Biomolecular Folding in Vacuo!!!(?). Proc. Natl. Acad. Sci. U.S.A. 1995, 92, 2426-2427.

4. Rodriguez-Cruz, S. E.; Klassen, J. S.; Williams, E. R. Hydration of Gas-Phase Gramicidin S $(\mathrm{M}+2 \mathrm{H})^{2+}$ Ions Formed by Electrospray: The Transition From Solution to Gas-Phase Structure. J. Am. Soc. Mass. Spectrom. 1997, 8, 565-568.

5. Woenckhaus, J.; Mao, Y.; Jarrold, M. F. Hydration of Gas Phase Proteins: Folded +5 and Unfolded +7 Charge States of Cytochrome c. J. Phys. Chem. B 1997, 101, 847-851.

6. (a) Jarrold, M. F. Unfolding, Refolding, and Hydration of Proteins in the Gas Phase. Acc. Chem. Res. 1999, 32, 360-367. (b) Jarrold, M. F. Peptides and Proteins in the Vapor Phase. Ann. Rev. Phys. Chem. 2000, 51 and Proteir

7. Lee, S.-W.; Freivogel, P.; Schindler, T.; Beauchamp, J. L. Freeze-Dried Biomolecules: FT-ICR Studies of the Specific Solvation of Functional Groups and Clathrate Formation Observed by the Slow Evaporation of Water from Hydrated Peptides and Model Compounds in the Gas Phase. J. Am. Chem. Soc. 1998, 120, 11758-11765.

8. Hoaglund-Hyzer, C. S.; Counterman, A. E.; Clemmer, D. E. Anhydrous Protein Ions. Chem. Rev. 1999, 99, 3037-3079.

9. Woenckhaus, J. Drift Time Mass Spectrometric Protein Hydration Experiments. Int. J. Mass Spectrom. 2002, 213, 9-24.

10. Breuker, K.; McLafferty, F. W. The Thermal Unfolding of Native Cytochrome $c$ in the Transition from Solution to Gas Phase Probed by Native Electron Capture Dissociation. Angew. Chem. Int. Ed. 2005, 44, 4911-4914.

11. Barran, P. E.; Polfer, N. C.; Campopiano, D. J.; Clarke, D. J.; LangridgeSmith, P. R. R.; Langley, R. J.; Govan, J. R. W.; Maxwell, A.; Dorin, J. R.; Millar, R. P.; Bowers, M. T. Is it Biologically Relevant to Measure the Structures of Small Peptides in the Gas-Phase? Int. J. Mass Spectrom. 2005, 240, 273-284.

12. (a) Chowdhury, S. K., Katta, V., Chait, B. T. Probing Conformational Changes in Proteins by Mass Spectrometry. J. Am. Chem. Soc. 1990, 112, 9012-9013. (b) Mirza, U. A.; Chait, B. T. Do Proteins Denature During Droplet Evolution in Electrospray Ionization? Int. J. Mass Spectrom. Ion Processes 1997, 162, 173-181.

13. Konermann, L.; Douglas, D. J. Equilibrium Unfolding of Proteins Monitored by Electrospray Ionization Mass Spectrometry: Distinguishing Two-State from Multi-State Transitions. Rapid Commun. Mass Spectrom. 1998, 12, 435-442.

14. (a) von Helden, G., Wyttenbach, T., Bowers, M. T. Conformation of Macromolecules in the Gas-Phase Use of Matrix-Assisted Laser-Desorption Methods in Ion Chromatography. Science 1995, 267, 1483-1485. (b) Wyttenbach, T.; von Helden, G.; Bowers, M. T. Gas-Phase Conformation of Biological Molecules: Bradykinin. J. Am. Chem. Soc. 1996, 118 , 8355-8364. 
15. Clemmer, D. E.; Hudgins, R. R.; Jarrold, M. F. Naked Protein Conformations: Cytochrome $c$ in the Gas Phase. J. Am. Chem. Soc. 1995, 117, 10141-10142.

16. Shelimov, K. B.; Jarrold, M. F. "Denaturation" and Refolding of Cytochrome $c$ in Vacuo. J. Am. Chem. Soc. 1996, 118, 10313-10314.

17. (a) Valentine, S. J.; Clemmer, D. E. H/D Exchange Levels of ShapeResolved Cytochrome $c$ Conformers in the Gas Phase. J. Am. Chem. Soc. 1997, 119, 3558-3566. (b) Valentine, S. J.; Anderson, J. G.; Ellington, A. D.; Clemmer, D. E. Disulfide-Intact and -Reduced Lysozyme in the Gas Phase: Conformations and Pathways of Folding and Unfolding. I. Phys. Chem. B 1997, 101, 3891-3900.

18. Jin, L.; Barran, P. E.; Deakin, J. A.; Lyon, M.; Uhrin, D. Conformation of Glycosaminoglycans by Ion Mobility Mass Spectrometry and Molecular Modeling. Phys. Chem. Chem. Phys. 2005, 7, 3464-3471.

19. Ruotolo, B. T.; Giles, K.; Campuzano, I.; Sandercock, A. M.; Bateman, R. H.; Robinson, C. V. Evidence for Macromolecular Protein Rings in the Absence of Bulk Water. Science 2005, 310, 1658-1661.

20. (a) Bernstein, S. L.; Wyttenbach, T.; Baumketnert, A.; Shea, J. E.; Bitan, G.; Teplow, D. B.; Bowers, M. T. Amyloid $\beta$-Protein: Monomer Structure and Early Aggregation States of A $\beta 42$ and its Pro(19) Alloform. J. Am. Chem. Soc. 2005, 127, 2075-2084. (b) Baumketner, A.; Bernstein, S. L.; Wyttenbach, T.; Bitan, G.; Teplow, D. B.; Bowers, M. T.; Shea, J. E. Amyloid $\beta$-Protein Monomer Structure: A Computational and Experimental Study. Prot. Sci. 2006, 15, 420-428

21. (a) Clemmer, D. E.; Jarrold, M. F. Ion Mobility Measurements and Their Applications to Clusters and Biomolecules. J. Mass Spectrom. 1997, 32, 577-592. (b) Wu, C.; Siems, W. F.; Klasmeier, J.; Hill, H. H. Separation of Isomeric Peptides Using Electrospray Ionization/High-Resolution Ion Mobility Spectrometry. Anal. Chem. 2000, 72, 391-395. (c) HoaglundHyzer, C. S.; Lee, Y. J.; Counterman, A. E.; Clemmer, D. E. Coupling Ion Mobility Separations, Collisional Activation Techniques, and Multiple Stages of MS for Analysis of Complex Peptide Mixtures. Anal. Chem. 2002, 74, 992-1006.

23. Koeniger, S. L; Merenbloom, S. I.; Valentine, S. J.; Jarrold, M. F.; Udseth, R. D.; Smith, R. D.; Clemmer, D. E. An IMS-IMS Analogue of MS-MS. Anal. Chem. 2006, 78, 4161-4174.

24. Koeniger, S. L.; Merenbloom, S. I.; Clemmer, D. E. Evidence for Many Resolvable Structures within Conformation Types of Electrosprayed Ubiquitin Ions. J. Phys. Chem. B 2006, 110, 7017-7021.

25. Merenbloom, S. I.; Koeniger, S. L.; Valentine, S. J.; Plasencia, M. D.; Clemmer, D. E. IMS-IMS and IMS-IMS-IMS/MS for Separating Peptide and Protein Fragment Ions. Anal. Chem. 2006, 78, 2802-2809.

26. Koeniger, S. L.; Merenbloom, S. I.; Sevugarajan, S.; Clemmer, D. E. Transfer of Structural Elements from Compact to Extended States in Unsolvated Ubiquitin. J. Am. Chem. Soc. 2006, 128, 11713-11719.

27. (a) Katta, V.; Chait, B. T. Conformational Changes in Proteins Probed by Hydrogen-Exchange Electrospray-Ionization Mass Spectrometry. Rapid Commun. Mass Spectrom. 1991, 5, 214-217. (b) Katta, V.; Chait, B. T. Hydrogen-Deuterium Exchange Electrospray-Ionization Mass-Spectrometry-A Method for Probing Protein Conformational Changes in Solution. J. Am. Chem. Soc.1993, 115, 6317-6321.

28. Loo, R. R. O.; Smith, R. D. Investigation of the Gas-Phase Structures of Electrosprayed Proteins Using Ion-Molecule Reactions. J. Am. Soc. Mass Spectrom. 1994, 5, 207-220.

29. Loo, J. A.; Quinn, J. P. Ryu, S. I.; Henry, K. D.; Senko, M. W.; McLafferty, F. W. High-Resolution Tandem Mass-Spectrometry of Large Biomolecules. Proc. Natl. Acad. Sci. U.S.A. 1992, 89, 286-289.

30. Senko, M. W.; Hendrickson, C. L.; Pasa-Tolic, L.; Marto, J. A.; White, F. M.; Guan, S. H.; Marshall, A. G. Electrospray Ionization Fourier Transform Ion Cyclotron Resonance at 9.4 T. Rapid Commun. Mass Spectrom. 1996, 10, 1824-1828.

31. Valentine, S. J.; Counterman, A. E.; Clemmer, D. E. Conformer-Dependent Proton-Transfer Reactions of Ubiquitin Ions. J. Am. Chem. Soc. Mass Spectrom. 1997, 8, 954-961.

32. Li, J.; Taraszka, J. A.; Counterman, A. E.; Clemmer, D. E. Influence of Solvent Composition and Capillary Temperature on the Conformations of Electrosprayed Ions: Unfolding of Compact Ubiquitin Conformers from Pseudonative and Denatured Solutions. Int. J. Mass Spectrom. 1999, 185/186/187, 37-47.

33. Covey, T.; Douglas, D. J. Collision Cross-Sections for Protein Ions. J. Am. Soc. Mass Spectrom. 1993, 4, 616-623.

34. Cox, K. A.; Julian, R. K., Jr.; Cooks, R. G.; Kaiser, R. E., Jr. Conformer Selection of Protein Ions by Ion Mobility in a Triple Quadrupole Mass-Spectrometer. J. Am. Chem. Soc. Mass Spectrom. 1994, 5, 127-136.

35. (a) Schnier, P. D., Gross, D. S., Williams, E. R. Electrostatic Forces and Dielectric Polarizability of Multiply Protonated Gas-Phase Cytochrome c Ions Probed by Ion/Molecule Chemistry. J. Am. Chem. Soc. 1995, 117, 6747-6757. (b) Gross, D. S.; Williams, E. R. Experimental Measurement of Coulomb Energy and Intrinsic Dielectric Polarizability of a Multiply Protonated Peptide Ion Using Electrospray Ionization Fourier-Transform Mass Spectrometry. I. Am. Chem. Soc. 1995, 117, 883-890. (c) Williams, E. R. Proton Transfer Reactivity of Large Multiply Charged Ions. J. Mass Spectrom. 1996, 31, 831-842.

36. (a) Cassady, C. J.; Wronka, J.; Kruppa, G. H.; Laukien, F. H. Deprotonation Reactions of Multiply Protonated Ubiquitin Ions. Rapid Commun.
Mass Spectrum. 1994, 8, 394-400. (b) Cassady, C. J.; Carr, S. R. Elucidation of Isomeric Structures for Ubiquitin $[\mathrm{M}+12 \mathrm{H}]^{12+}$ Ions Produced by Electrospray Ionization Mass Spectrometry. J. Mass Spectrom. 1996, 31, 247-254. (c) Zhang, X.; Cassady, C. J. Apparent Gas-Phase Acidities of Multiply Protonated Peptide Ions: Ubiquitin, Insulin B, and Renin Substrate. J. Am. Soc. Mass Spectrom. 1996, 7, 1211-1218.

37. Freitas, M. A.; Hendrickson, C. L.; Emmett, M. R.; Marshall, A. G Gas-Phase Bovine Ubiquitin Cation Conformations Resolved by GasPhase Hydrogen/Deuterium Exchange Rate and Extent. Int. J. Mass Spectrom. 1999, 187, 565-575.

38. Evans, S. E.; Lueck, N.; Marzluff, E. M. Gas Phase Hydrogen/Deuterium Exchange of Proteins in an Ion Trap Mass Spectrometer. Int. J. Mass Spectrom. 2003, 222, 175-187.

39. Geller, O.; Lifshitz, C. A Fast Flow Tube Study of Gas Phase H/D Exchange of Multiply Protonated Ubiquitin. J. Phys. Chem. A 2005, 109, 2217-2222.

40. Robinson, E. W.; Williams, E. R. Multidimensional Separations of Ubiquitin Conformers in the Gas Phase: Relating Ion Cross Sections to H/D Exchange Measurements. J. Am. Soc. Mass Spectrom. 2005, 16, 1427-1437.

41. Badman, E. R.; Hoaglund-Hyzer, C. S.; Clemmer, D. E. Gas-Phase Separations of Protein and Peptide Ion Fragments Generated by Collision-Induced Dissociation in an Ion Trap. Anal. Chem. 2001, 73, 60006007.

42. Myung, S.; Badman, E.; Lee, Y. J.; Clemmer, D. E. Structural Transitions of Electrosprayed Ubiquitin Ions Stored in an Ion Trap over $10 \mathrm{~ms}$ to 30 s. J. Phys. Chem. A 2002, 106, 9976-9982.

43. (a) Purves, R. W.; Barnett, D. A.; Ells, B; Guevremont, R. Investigation of Bovine Ubiquitin Conformers Separated by High-Field Asymmetric Waveform Ion Mobility spectrometry: Cross Section Measurements Using Energy-Loss Experiments with a Triple Quadrupole Mass Spectrometer I. Am. Soc. Mass Spectrom. 2000, 11, 738-745. (b) Purves, R. W. Barnett, D. A.; Ells, B.; Guevremont, R. Elongated Conformers of Charge States +11 to +15 of Bovine Ubiquitin Studied Using. ESI-FAIMS-MS J. Am. Soc. Mass Spectrom. 2001, 12, 894-901.

44. Shvartsburg, A. A., Fumin, L., Tang, K., Smith, R. D. Characterizing the Structures and Folding of Free Proteins Using 2-D Gas-Phase Separations: Observation of Multiple Unfolded Conformers. Anal. Chem. Articles ASAP, DOI: 10.1021/ac060283z.

45. Purves, R. W.; Barnett, D. A.; Guevremont, R. Separation of Protein Conformers Using Electrospray-High Field Asymmetric Waveform Ion Mobility Spectrometry-Mass Spectrometry. Int. J. Mass Spectrom. 2000, 197, 163-177.

46. Wyttenbach, T.; Kemper, P. R.; Bowers, M. T. Design of a New Electrospray Ion Mobility Mass Spectrometer. Int. J. Mass Spectrom. 2001, 212, 13-23.

47. Badman, E.; Myung, S.; Clemmer. D. E. Evidence for Unfolding and Refolding of Gas Phase Cystochrome $c$ Ions in a Paul Trap. J. Am. Soc. Mass Spectrom. 2005, 16, 1493-1497.

48. McLuckey, S. A.; Wells, J. M.; Stephenson, J. L.; Goeringer, D. E. Novel Quadrupole Ion Trap Methods for Characterizing the Chemistry of Gaseous Macro-Ions. Int. J. Mass Spectrom. 2000, 200, 137-161.

49. Rockwood, A. L.; Busman, M.; Smith, R. D. Coulombic Effects in the Dissociation of Large Highly Charged Ions. Int. J. Mass Spectrom. 1991, 111, 103-129.

50. Gross, D. S.; Schnier, P. D.; Rodriguez-Cruz, S. E.; Fagerquist, C. K.; Williams, E. R. Conformations and Folding of Lysozyme Ions in Vacuo. Proc. Natl. Acad. Sci. U.S.A. 1996, 93, 3143-3148.

51. Mason, E. A.; McDaniel; E. W. Transport Properties of Ions in Gases; Wiley: New York, 1988, p 276.

52. Badman, E. R.; Hoaglund-Hyzer, C. S.; Clemmer, D. E. Dissociation of Different Conformations of Ubiquitin Ions. J. Am. Soc. Mass Spectrom. 2002, 13, 719-723.

53. Edman, L.; Mets, U.; Rigler, R. Conformational Transitions Monitored for Single Molecules in Solution. Proc. Natl. Acad. Sci. U.S.A. 1996, 93, 6710-6715.

54. Kellermayer, M. S. Z.; Smith, S. B.; Granzier, H. L.; Bustamante, C. Folding-Unfolding Transitions in Single Titin Molecules Characterized with Laser Tweezers. Science 1997, 276, 1112-1116.

55. Lu, H. P.; Xun, L.Y.; Xie, X. S. Single-Molecule Enzymatic Dynamics. Science 1998, 282, 1877-1882.

56. (a) Ha, T. J.; Ting, A. Y.; Liang, J.; Caldwell, W. B.; Deniz, A. A.; Chemla, D. S.; Schultz, P. G.; Weiss, S. Single-Molecule Fluorescence Spectroscopy of Enzyme Conformational Dynamics and Cleavage Mechanism. Proc. Natl. Acad. Sci. U.S.A. 1999, 96, 893->898. (b) Deniz, A. A.; Laurence, T. A.; Beligere, G. S.; Dahan, M.; Martin, A. B.; Chemla, D. S.; Dawson, P. E.; Schultz, P. G.; Weiss, S. Single-Molecule Protein Folding: Diffusion Fluorescence Resonance Energy Transfer Studies of the Denaturation of Chymotrypsin Inhibitor 2. Proc. Natl. Acad. Sci. U.S.A. 2000, 97, 5179-5184.

57. Schuler, B.; Lipman, E. A.; Eaton, W. A. Probing the Free-Energy Surface for Protein Folding with Single-Molecule Fluorescence Spectroscopy. Nature 2002, 419, 743-747.

58. Fernandez, J. M.; Li, H. B. Force-Clamp Spectroscopy Monitors the Folding Trajectory of a Single Protein. Science 2004, 303, 1674-1678. 